\title{
Theranostics
}

Review

$2011 ; 1: 149-153$

\section{Integrin Imaging to Evaluate Treatment Response}

\author{
Matthew Morrison ${ }^{1 凶}$ and Alan Cuthbertson ${ }^{2}$ \\ 1. GE Healthcare MDx Research, The Grove Centre, Amersham, HP7 9LL, U.K. \\ 2. GE Healthcare MDx Research, Nycoveien 2, NO-0401 Oslo, Norway
}

Corresponding author: Dr. Matthew Morrison, GE Healthcare MDx Research, The Grove Centre, Amersham, HP7 9LL, U.K; Tel: 0044 (0)1494 545112; E-mail: matthew.morrison@ge.com

(c) Ivyspring International Publisher. This is an open-access article distributed under the terms of the Creative Commons License (http://creativecommons.org/ licenses/by-nc-nd/3.0/). Reproduction is permitted for personal, noncommercial use, provided that the article is in whole, unmodified, and properly cited.

Published: 2011.02.11

\begin{abstract}
Despite the recent development of various radiolabelled RGD peptides for imaging the $\alpha v \beta 3$ integrin receptor, relatively little attention has been focused on the ability of these radiotracers to monitor changes in tumour vascularity following treatment with anti-tumour therapies. Here we describe the favourable in vivo kinetics and tumour targeting properties of several novel radiolabeled RGD containing peptides that have the ability to monitor tumour vascularity non-invasively in a variety of preclinical tumor models. These tracers may reveal important information when assessing the impact of anti-tumour therapies, in particular those that predominantly target tumour blood vessels. Consequently, these radiolabelled RGD targeting agents represent a useful approach to quantify tumor vasculature, and when used alone or in combination with additional functional imaging modalities, should enhance our mechanistic understanding of how novel therapeutic strategies impact upon tumors.
\end{abstract}

Key words: RGD peptide; Radiolabeling; Cancer treatment response; Tumor vasculature.

\section{INTRODUCTION}

A number of novel therapeutic strategies are currently being examined clinically which aim to modify tumour vasculature function, growth and survival [1]. These include inhibition of vascular endothelial growth factor-A (VEGF) signaling [1] (a key regulator of tumour angiogenesis, vascular permeability and neovascular survival), use of vascular disrupting agents (VDAs) such as the tubulin-depolymerising agent combretastatin A-4 [2], or examination of low dose cytotoxic chemotherapy [3]. To monitor the effect of VEGF signaling inhibitors or VDAs, Magnetic Resonance Imaging (MRI) or Computerised Tomography (CT), and in particular Dynamic Contrast Enhanced MRI (DCE-MRI) and CT (DCE-CT) have been employed successfully to provide evidence of an effect on tumour blood flow, blood volume and permeability [4-5]. However, an obvious current limitation to being able to understand the full activity of approaches that target the tumour vasculature, is the lack of a non-invasive imaging method to quantify changes in tumour vascularity in cancer patients [6]. This may become of greater importance as alternative (i.e. non-VEGF / VDA) therapies are progressed clinically [1], since these may have more subtle and less predictable acute effects on tumour haemodynamics.

Integrins are composed of a family of heterodimeric glycoproteins involved in cell-cell and cell-matrix interactions [7]. They consist of $\alpha$ and $\beta$ subunits that form noncovalent $\alpha \beta$ heterodimers [8] and are the major receptors by which cells attach to the extracellular matrix (ECM). The $\alpha v \beta 3$ integrin, 
which is preferentially expressed on proliferating endothelial cells associated with neovascularisation in both malignant tumours and normal tissue, but not in quiescent blood vessels [9-10], has been identified as a target for imaging neovasculature. Others have shown previously [11-13] that RGD peptides can serve as a targeting biomolecule to carry a range of radionuclides (e.g., ${ }^{18} \mathrm{~F},{ }^{99 \mathrm{mTc}}$ and ${ }^{64} \mathrm{Cu}$ ) to the $\alpha \mathrm{v} \beta 3$ integrin. Thus, targeting the $\alpha v \beta 3$ integrin receptor with $\left[{ }^{18} \mathrm{~F}\right]$ fluciclatide (formerly known as [18F]AH111585) could provide us with a tool to examine the effect of vascular modulation therapies directly, without having to rely on consequential changes in blood perfusion and permeability. This may be particularly pertinent to examining therapies that specifically target the tumour vasculature, given that their activity may not lead to a substantial tumour mass/volume reduction soon after therapy [14-15]. Hence conventional measurements of response may be insensitive or markedly delayed even when there is a significant therapeutic effect. Direct clinical imaging of tumour neovasculature, could therefore afford the opportunity to optimise dose-selection during early examination of a novel anti-vascular therapy.

Previously, quantification of tumour vasculature has been assessed in surgical specimens or preclinical samples using immunohistochemical methods. However, not only is there potential variability in the measurement of MVD [15], dependent upon the marker (e.g. CD31, CD105) or endpoint used (e.g. Chalkey Counts, whole tumour image analysis, "hot-spot" analysis), but such assays are not practical in clinical trials. The ethical and physical limitations of serial invasive procedures and the inherent anatomic and physiologic heterogeneity of tumours is likely to necessitate multiple samples being taken at any given time-point [16]. Clearly a non-invasive method for assessing tumour vascularity could obviate these technical challenges.

$\left.{ }^{[18} \mathrm{F}\right]$ fluciclatide is a novel cyclic RGD-based radioligand for $\alpha_{v} \beta_{3} / \alpha_{v} \beta_{5}$, an analogue of a peptide pharmacophore isolated from a phage display library [17]. The original peptide sequence (RGD-4C) was optimised extensively to improve in vivo stability and increase plasma half-life [18] whilst maintaining high affinity for $\alpha_{v} \beta_{3} / \alpha_{v} \beta_{5}$ : the observed $K_{i}$ for the cold compound (AH111585) was calculated to be $10.2 \mathrm{nM}$ in competition with radioactive 125 I-Echistatin, an RGD-containing peptide isolated from snake venom.

In addition, the fact that $\left[{ }^{18} \mathrm{~F}\right]$ fluciclatide is able to target neovasculature via the $\alpha_{\mathrm{v}} \beta_{3} / \alpha_{\mathrm{v}} \beta_{5}$ receptors expressed on endothelial cells is supported by the work of Pasqualini et al., who demonstrated that tu- mour vessels were the structural elements most targeted by the RGD sequence [19].

\section{USE OF RGD-BASED RADIOLIGANDS FOR THERAPY MONITORING}

Although several groups have developed radiolabelled RGD compounds which have shown promise in terms of tumour uptake and tumour to background ratios [20-22], relatively little attention has been focused on the ability of these radiotracers to monitor the response of tumors to therapies that target the vasculature, the most likely clinical use of these imaging agents. One exception is the work by Jung et al. [23] who have demonstrated that paclitaxel therapy (an anti-microtubule agent commonly used in the treatment of breast and NSCLC [24]) resulted in a decreased LLC uptake of a $99 \mathrm{mTc}$-labelled glucosamino RGD containing peptide. The authors stated that these data support the use of the radiolabelled RGD peptide for monitoring response to anti-angiogenic therapy.

We have also demonstrated the utility of $\left.{ }^{[18} \mathrm{F}\right]$ fluciclatide in determining changes in the level of tumor vascularity following treatment with the same chemotherapeutic agent, paclitaxel [25]. However, one important point to note is that in our study, paclitaxel was used at lower doses to that used by Jung et al.; animals were dosed at 5 and $10 \mathrm{mg} / \mathrm{kg}$. At these low doses, paclitaxel is reported to cause an anti-angiogenic effect without significant tumour shrinkage [26].

In order to acquire comparison data to $\left[{ }^{18} \mathrm{~F}\right]$ fluciclatide, we co-injected $\left[{ }^{14} \mathrm{C}\right]-\mathrm{FDG}$ with $\left.{ }^{[18} \mathrm{F}\right]$ fluciclatide post paclitaxel therapy in order to generate data with an imaging agent (when ${ }^{18} \mathrm{~F}$ radiolabelled) that is used extensively in the clinic for tumour imaging [27]. ${ }^{18}$ Ffluciclatide tumour uptake was more sensitive to paclitaxel therapy, since a reduction in uptake was seen while $\left[{ }^{14} \mathrm{C}\right]$-FDG uptake remained unchanged (Fig. 1). The reduction in $\left.{ }^{[18} \mathrm{F}\right]$ fluciclatide uptake was matched by a decrease in the tumour h-MVD: with low dose paclitaxel, a $20 \%$ decrease in MVD was detected when compared to a separate group of vehicle control treated LLC tumors. This demonstrates that $\left[{ }^{18} \mathrm{~F}\right]$ fluciclatide is able to detect vascular changes that occur through the use of paclitaxel. It is postulated that the slight increase in uptake with $\left[{ }^{14} \mathrm{C}\right]-\mathrm{FDG}$ in the paclitaxel treated animals reflected the inflammatory flare typically seen with [18F]-FDG after therapy [28], but this was not significant.

Following on from the promising results seen with paclitaxel and $\left[{ }^{18} \mathrm{~F}\right]$ fluciclatide, we were interested to understand the impact of more novel an- 
ti-angiogenic therapies on $\left[{ }^{18} \mathrm{~F}\right]$ fluciclatide uptake preclinically. Initially we assessed the impact of ZD4190, a small molecular VEGFR-2 tyrosine kinase inhibitor [29]. ZD4190 was administered orally (100 $\mathrm{mg} / \mathrm{kg}$ ) to mice bearing Calu- 6 tumors after tumor establishment on 3 occasions following a baseline PET scan with a follow up PET scan on day 3.

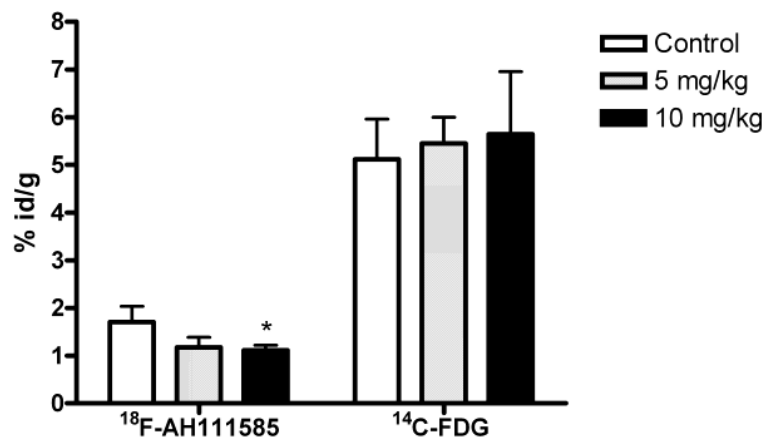

Figure I. Inhibition of $\left[{ }^{18} \mathrm{~F}\right]$ fluciclatide uptake by low dose paclitaxel ( 5 and $10 \mathrm{mg} / \mathrm{kg}$ ) therapy in the mouse Lewis Lung tumour model ( 120 minutes p.i. of $\left[{ }^{18} \mathrm{~F}\right]$ fluciclatide, day 15 post LLC cell inoculation). At the same paclitaxel dose level, no effect was observed on $\left[{ }^{14} \mathrm{C}\right]-$ FDG uptake. Values are expressed in \%id/g and presented as Mean (SD) of 2 experiments $(n=4)$. $* P<0.05$ compared with corresponding control group.

As can be seen (Fig. 2), the uptake of $\left[{ }^{18} \mathrm{~F}\right]$ fluciclatide in Calu-6 tumors was reduced significantly with 3 doses of ZD4190, with an average reduction in the percentage injected dose per gram (\%ID/g) across the 10 animals examined of $31.8 \% \pm$ $4.6 \%$ (based on the imaging region-of-interest (ROI) data). Skeletal muscle was used as a reference tissue for ROI analysis, and the data demonstrate that muscle uptake was maintained before and after therapy, indicating that the tumor decrease seen with ZD4190 therapy was specific. Comparison with the vehicle control animals demonstrates that over the same 3-day dosing period, the Calu-6 tumor uptake of ${ }^{18}$ Ffluciclatide increased from the pre to the post treatment image, with an average increase of $26.9 \% \pm$ $9.4 \%$. Statistical analysis of the treated and control groups demonstrated a clear difference at the $\mathrm{P}<0.01$ level (determined via $t$ test).

Figure 3 shows representative fused small animal PET and micro-CT images for ZD4190-treated and vehicle control animals. The before and after images show decreased $\left[{ }^{18} \mathrm{~F}\right]$ fluciclatide uptake (with ZD4190 therapy) and an increased [ $\left.{ }^{18} \mathrm{~F}\right]$ fluciclatide uptake (with vehicle control) before and after therapy.

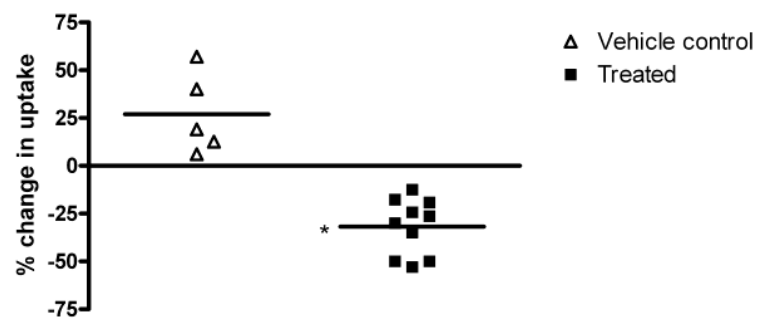

Figure 2. Summarized data demonstrating the percentage change in uptake of $\left[{ }^{18} \mathrm{~F}\right]$ fluciclatide in Calu- 6 tumors, pre and post treatment with 3 doses ZD4190 $(100 \mathrm{mg} / \mathrm{kg})$ or vehicle control. Data presented for individual animals. $* \mathrm{P}<0.0 \mathrm{l}$ compared to vehicle control group.

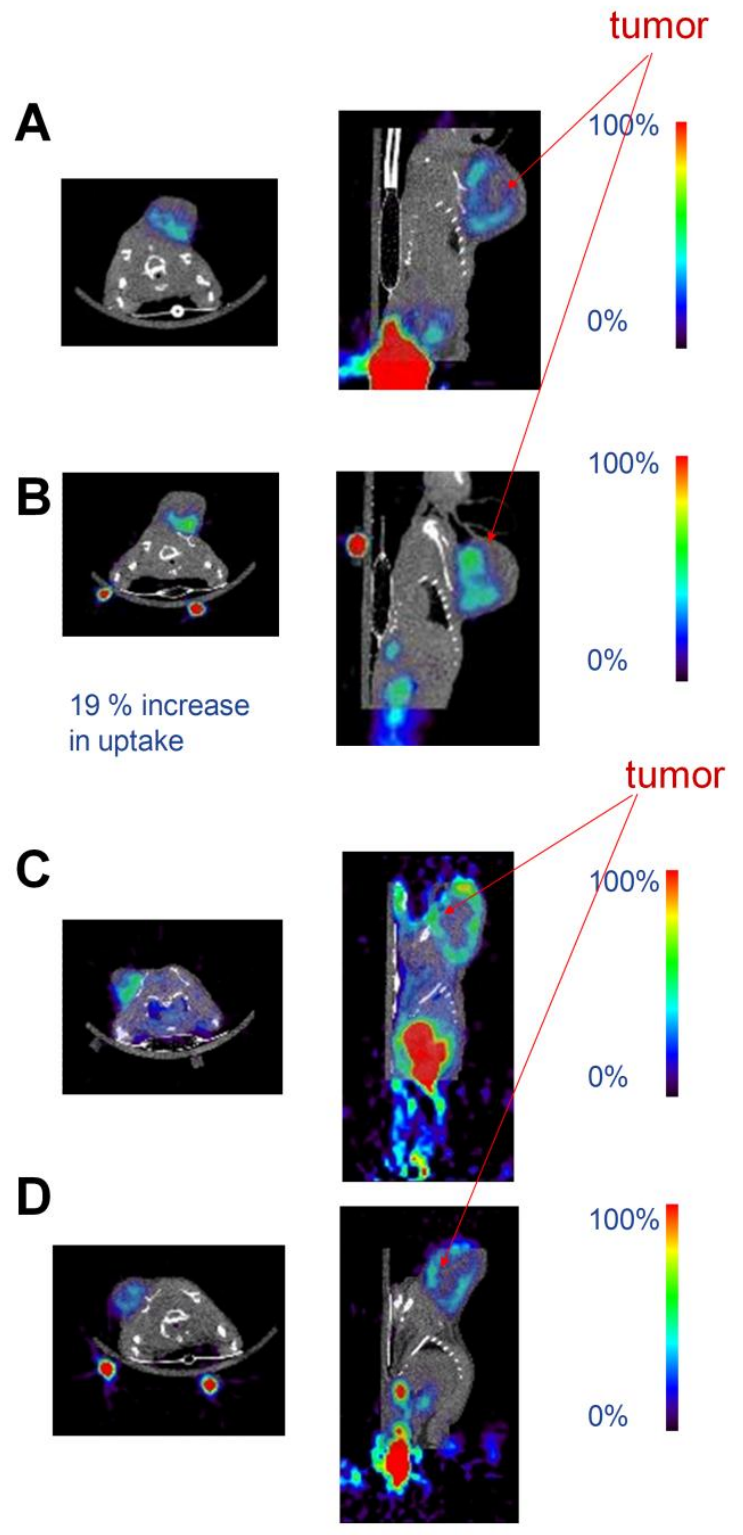

Figure 3. Representative co-registered microPET and microCT images demonstrating $\left[{ }^{18} \mathrm{~F}\right]$ fluciclatide uptake at 120 min in the Calu-6 tumour model pre (a) and post (b) 
administration of 3 doses of vehicle control or pre (c) and post (d) 3 doses of ZD4190 (100 mg/kg). Contrast is clearly seen in the Calu-6 tumours located on the left shoulder region in both the ZD4190 treated and vehicle control animals. The only additional higher activity concentration was found in the bladder. In addition, ${ }^{22} \mathrm{Na}$ fiducial markers can be seen located on the base of the imaging bed (used for PET/CT co-registration). ROI analysis for tumour uptake pre ZD4 190 therapy was $1.7 \% \mathrm{ld} / \mathrm{g}$ decreasing to $1.1 \% \mathrm{ld} / \mathrm{g}$ post ZD4 190 therapy. Muscle uptake was maintained at 0.5 $\% \mathrm{ld} / \mathrm{g}$ in pre and post ZD4190 therapy images. For animals treated with vehicle control alone, ROI analysis for tumour uptake pre-vehicle control was $2.1 \% \mathrm{ld} / \mathrm{g}$ increasing to 2.5 $\%$ Id/g post-therapy. Muscle uptake was maintained at 0.3 $\% \mathrm{ld} / \mathrm{g}$ in pre and post therapy images.

We have also carried out additional preclinical studies using sunitinib (Sutent, Pfizer, Inc.), an oral, small molecule tyrosine kinase inhibitor with nanomolar affinity against the VEGFR and PDGFR tyrosine kinases [30]. U87MG bearing animals were dosed with sunitinib or vehicle alone using a five-day-on/two-day-off treatment cycle previously described [31]. In a similar way to that described for ZD4190, sunitinib was able to significantly reduce the uptake of $\left[{ }^{18} \mathrm{~F}\right]$ fluciclatide compared to vehicle control treated U87MG xenograft tumors from day 2 onward post therapy initiation.

The Weber group has also recently published data assessing whether their radiolabelled RGD agent $\left(\left[{ }^{64} \mathrm{Cu}\right] D O T A-c y c l o-(R G D f K)\right)$ can be used for monitoring response to the Src family kinase inhibitor dasatinib [32]. Using U87MG xenograft tumors dosed daily over 3 days with 72 or $95 \mathrm{mg} / \mathrm{kg}$ dasatinib or vehicle they found that the uptake of [ $\left.{ }^{64} \mathrm{Cu}\right]$ DOTA-cyclo-(RGDfK) was significantly reduced in the $95 \mathrm{mg} / \mathrm{kg}$ dose group by $59 \%$ compared to control animals. The group also reported that in contrast to the decrease seen with $\left[{ }^{64} \mathrm{Cu}\right.$ ]DOTA-cyclo-(RGDfK) in dasatinib treated tumours, ${ }^{18}$ F-FDG uptake showed no significant reduction, which matches the data we generated using $\left[{ }^{14} \mathrm{C}\right]$ FDG with paclitaxel therapy.

In conclusion, the data emerging from our own studies and those of Jung et al. and Dumont et al. suggest that radiolabelled RGD agents with favorable biodistribution properties do allow the non-invasive assessment of tumour vascularity and response to treatments that have an effect on the tumour vascular compartment. Additionally preliminary clinical studies with our tracer, [18F]fluciclatide have already proven that this radioligand can successfully image metastatic breast cancer lesions [33]: 7 patients with a total of 18 tumors detectable by computer tomography $(\mathrm{CT})$ were imaged with [18F]fluciclatide PET, and all tumors were visible. Similar work has also been published using other radiolabelled RGD agents, in, for example, malignant melanoma [34].

Consequently, these radiolabelled RGD targeting agent represents a useful approach to quantify tumour vasculature, and when used alone or in combination with additional functional imaging modalities, should enhance our mechanistic understanding of how novel therapeutic strategies impact upon tumors.

\section{ACKNOWLEDGMENTS}

We would like to thank Pfizer and AstraZeneca for their kind contribution by supplying the sunitinib and ZD4190 compounds respectively for use in these studies.

\section{Conflict of Interest}

The authors have declared that no conflict of interest exists.

\section{References}

1. Folkman J. Angiogenesis: an organizing principle for drug discovery? Nat Rev Drug Discov. 2007;6:273-86.

2. Hinnen P, Eskens FA. Vascular disrupting agents in clinical development. Br J Cancer. 2007;96:1159-65.

3. Munoz R, Shaked Y, Bertolini F, Emmenegger U, Man S, Kerbel RS. Anti-angiogenic treatment of breast cancer using metronomic low-dose chemotherapy. Breast. 2005;14:466-79.

4. Bradley DP, Tessier JL, Checkley D, Kuribayashi H, Waterton JC, Kendrew J, et al. Effects of AZD2171 and vandetanib (ZD6474, Zactima) on haemodynamic variables in an SW620 human colon tumour model: an investigation using dynamic contrast-enhanced MRI and the rapid clearance blood pool contrast agent, P792 (gadomelitol). NMR Biomed. 2008;21:42-52.

5. Daldrup-Link HE, Simon GH, Brasch RC. Imaging of tumor angiogenesis: current approaches and future prospects. Curr Pharm Des. 2006;12:2661-72.

6. McDonald DM, Teicher BA, Stetler-Stevenson W, Ng SS, Figg WD, Folkman J, et al. Report from the society for biological therapy and vascular biology faculty of the NCI workshop on angiogenesis monitoring. J Immunother. 2004;27:161-75.

7. Horton MA. The av $\beta 3$ integrin "vitronectin receptor". Int J Biochem Cell Biol. 1997;29:721-5.

8. Hynes RO. Integrins: versatility, modulation, and signaling in cell adhesion. Cell. 1992;69:11-25.

9. Eliceiri BP, Cheresh DA. The role of alphav integrins during angiogenesis: insights into potential mechanisms of action and clinical development. J Clin Invest. 1999;103:1227-30.

10. Brooks PC, Montgomery AM, Rosenfeld M, Reisfeld RA, Hu T, Klier $\mathrm{G}$, et al. Integrin av $\beta 3$ antagonists promote tumor regression by inducing apoptosis of angiogenic blood vessels. Cell. 1994;79:1157-64.

11. Beer AJ, Niemeyer M, Carlsen J, Sarbia M, Nahrig J, Watzlowik $P$, et al. Patterns of $\alpha v \beta 3$ expression in primary and metastatic human breast cancer as shown by ${ }^{18} \mathrm{~F}-$ Galacto-RGD PET. J Nucl Med. 2008;49:255-9. 
12. Chen X, Tohme M, Park R, Hou Y, Bading JR, Conti PS. Micro-PET imaging of av $\beta 3$-integrin expression with ${ }^{18}$ F-labeled dimeric RGD peptide. Mol Imaging. 2004;3:96-104.

13. Haubner RH, Wester HJ, Weber WA, Schwaiger $M$. Radiotracer-based strategies to image angiogenesis. Q J Nucl Med. 2003;47:189-99.

14. Hahnfeldt P, Panigrahy D, Folkman J, Hlatky L. Tumor development under angiogenic signaling: a dynamical theory of tumor growth, treatment response, and postvascular dormancy. Cancer Res. 1999;59:4770-5.

15. Hlatky L, Hahnfeldt P, Folkman J. Clinical application of antiangiogenic therapy: microvessel density, what it does and doesn't tell us. J Natl Cancer Inst. 2002;94:883-93.

16. Gerber HP, Ferrara N. Pharmacology and pharmacodynamics of bevacizumab as monotherapy or in combination with cytotoxic therapy in preclinical studies. Cancer Res. 2005;65:671-80.

17. Pasqualini R, Koivunen E, Ruoslahti E. A peptide isolated from phage display libraries is a structural and functional mimic of an RGD-binding site on integrins. J Cell Biol. 1995;130:1189-96.

18. Indrevoll B, Kindberg GM, Solbakken M, Bjurgert E, Johansen JH, Karlsen H, et al. NC-100717: a versatile RGD peptide scaffold for angiogenesis imaging. Bioorg Med Chem Lett. 2006;16:6190-3.

19. Pasqualini R, Koivunen E, Ruoslahti E. Alpha v integrins as receptors for tumor targeting by circulating ligands. Nat Biotechnol. 1997;15:542-6.

20. Liu S, Hsieh WY, Jiang Y, Kim YS, Sreerama SG, Chen X, et al. Evaluation of a $99 \mathrm{~m} T \mathrm{~T}-$ labeled cyclic RGD tetramer for noninvasive imaging integrin av $\beta 3$-positive breast cancer. Bioconjug Chem. 2007;18:438-46.

21. Haubner R. Alphavbeta3-integrin imaging: a new approach to characterise angiogenesis? Eur J Nucl Med Mol Imaging. 2006;33 (Suppl 1):54-63.

22. Beer AJ, Niemeyer M, Carlsen J, Sarbia M, Nahrig J, Watzlowik $\mathrm{P}$, et al. Patterns of av $\beta 3$ Expression in Primary and Metastatic Human Breast Cancer as Shown by ${ }^{18} \mathrm{~F}-$ Galacto-RGD PET. J Nucl Med. 2008;49:255-9.

23. Jung KH, Lee KH, Paik JY, Ko BH, Bae JS, Lee BC, et al. Favorable biokinetic and tumor-targeting properties of 99mTc-labeled glucosamino RGD and effect of paclitaxel therapy. J Nucl Med. 2006;47:2000-7.

24. Horwitz SB. Taxol (paclitaxel): mechanisms of action. Ann Oncol. 1994;5 (Suppl 6):S3-6.

25. Morrison MS, Ricketts SA, Barnett J, Cuthbertson A, Tessier J, Wedge SR. Use of a novel Arg-Gly-Asp radioligand, ${ }^{18} \mathrm{~F}-\mathrm{AH} 111585$, to determine changes in tumor vascularity after antitumor therapy. J Nucl Med. 2009;50:116-22.

26. Lau DH, Xue L, Young LJ, Burke PA, Cheung AT. Paclitaxel (Taxol): an inhibitor of angiogenesis in a highly vascularized transgenic breast cancer. Cancer Biother Radiopharm. 1999;14:31-6.

27. Juweid ME. Utility of positron emission tomography (PET) scanning in managing patients with Hodgkin lymphoma. Hematology Am Soc Hematol Educ Program. 2006;:259-65.

28. Mortimer JE, Dehdashti F, Siegel BA, Trinkaus K, Katzenellenbogen JA, Welch MJ. Metabolic flare: indicator of hormone responsiveness in advanced breast cancer. J Clin Oncol. 2001;19:2797-803.

29. Wedge SR, Ogilvie DJ, Dukes M, Kendrew J, Curwen JO, Hennequin LF, et al. ZD4190: an orally active inhibitor of vascular endothelial growth factor signaling with broad-spectrum antitumor efficacy. Cancer Res. 2000;60:970-5.

30. Westermark B, Heldin CH, Nister M. Platelet-derived growth factor in human glioma. Glia. 1995;15:257-63.

31. de Bouard S, Herlin P, Christensen JG, Lemoisson E, Gauduchon P, Raymond E, et al. Antiangiogenic and anti-invasive effects of sunitinib on experimental human glioblastoma. Neuro Oncol. 2007;9:412-23.

32. Dumont RA, Hildebrandt I, Su H, Haubner R, Reischl G, Czernin JG, et al. Noninvasive imaging of $\operatorname{av} \beta 3$ function as a predictor of the antimigratory and antiproliferative effects of dasatinib. Cancer Res. 2009;69:3173-9.

33. Kenny LM, Coombes CR, Oulie I, Contractor K, Miller M, Spinks T, et al. The RGD radioligand $\left[{ }^{18} \mathrm{~F}\right] \mathrm{AH} 111585$ : A novel probe for imaging $\alpha v \beta 3$ and $\operatorname{av} \beta 5$ receptor levels by positron emission tomography in breast cancer patients. J Nucl Med. 2008;49:879-86.

34. Haubner R, Weber WA, Beer AJ, Vabuliene E, Reim D, Sarbia $\mathrm{M}$, et al. Noninvasive visualization of the activated $\operatorname{av} \beta 3$ integrin in cancer patients by positron emission tomography and $\left.{ }^{18} \mathrm{~F}\right]$ Galacto-RGD. PLoS Med. 2005;2:e70. 